\title{
23. Wissenschaftliche Jahrestagung der Deutschen Gesellschaft für Sozialmedizin und Prävention
}

Dieses Heft ist der 23. Wissenschaftlichen Jahrestagung der Deutschen Gesellschaft für Sozialmedizin und Prävention gewidmet. Die 23. Jahrestagung fand im September 1987 in Augsburg statt und befasste sich mit dem Thema «Herz-Kreislaufkrankheiten - eine medizinische und soziale Aufgabe». Namhafte Schweizer Kollegen haben an der Jahrestagung teilgenommen und wesentlich zu ihrem Gelingen beigetragen.

Ein Höhepunkt der Jahrestagung war die Verleihung der Salomon-Neumann-Medaille an Professor F. H. Epstein, Zürich, für seine grossen Verdienste um die Epidemiologie und Prävention der Herz-Kreislaufkrankheiten.

Das Thema Herz-Kreislaufkrankheiten war gewählt worden, weil diese Krankheitsgruppe für die Krankheitslandschaft der Bundesrepublik Deutschland (BRD) von besonderer Bedeutung ist. Wir alle wissen, dass in der BRD etwa die Hälfte aller Todesfälle auf diese Krankheitsgruppe zurückzuführen ist. Wenn man nur die sogenannten vorzeitigen Todesfälle betrachtet und sich auf Männer der Altersgruppe 40-69 bezieht, ersieht man, dass die Herz-Kreislaufkrankheiten mit einem Anteil von $42 \%$ an diesen Todesfällen beteiligt sind. Der entsprechende Anteil der ischämischen Herzkrankheiten liegt bei ca. $25 \%$. Für Frauen sehen die entsprechenden Zahlen nicht ganz so gravierend aus.

Die Bedeutung der Herz-Kreislaufkrankheiten für den einzelnen und für dic Gesellschaft wird noch klarer, wenn wir über die Betrachtung der Sterblichkeit hinausgehen und auch Morbidität und Behinderung in unsere Überlegungen miteinbeziehen. In der BRD lag z. B. 1984 der Anteil der Erwerbs- und Berufsunfähigkeitsrenten, die wegen Herz-Kreislaufkrankheiten gewährt wurden, bei $35 \%$. Dieser Prozentsatz bezieht sich auf Männer und Frauen, Arbeiter und Angestellte. Auf der Häufigkeitsskala der Berentungsursachen stehen die ischämischen Herzkrankheiten an erster Stelle; etwa ein Drittel dieser Renten wird vor dem 54. Lebensjahr gewährt.

Diese wenigen Zahlen machen deutlich, dass die Herz-
Kreislaufkrankheiten grossen Einfluss auf Sterblichkeit, vorzeitige Sterblichkeit, Morbidität und Behinderung in unserer Bevölkerung haben.

Die Prävention der Herz-Kreislaufkrankheiten ist offensichtlich nicht nur eine medizinische, sondern weit mehr noch eine gesellschaftliche Aufgabe. Gerade in der BRD treffen wir bei unseren Bemühungen um eine gesündere Lebensweise der Bevölkerung auf grosse Widerstände. Fast jede von uns propagierte Veränderung tangiert die Interessen bestimmter Gruppen. Ich denke hier nicht nur an die Zigarettenindustrie, sondern z. B. auch an die deutsche Landwirtschaft, welche mit Annoncen im Deutschen Ärzteblatt das Ziel verfolgt, u. a. die Ergebnisse des Lipid Research Clinics Coronary Primary Prevention Trial (LRCCPPT) in Frage zu stellen und den kausalen Zusammenhang zwischen Senkung des Cholesterinspiegels und Sinken der Mortalität an Herzinfarkt zu leugnen. Diese beiden Beispiele machen deutlich, welche enormen Anstrengungen notwendig sind, um in der Bundesrepublik die Ergebnisse wichtiger epidemiologischer Studien zum Wohle der Bevölkerung in die Praxis umzusetzen.

Auf der Augsburger Jahrestagung wurde in über 100 Vorträgen und Postern vorwiegend zu Themen der Epidemiologie, Prävention und Rehabilitation der koronaren Herzkrankheit (KHK) und der Hypertonie Stellung genommen. Auch das WHO MONICA Projekt spielte eine wichtige Rolle. Prominente Wissenschaftler nahmen auch zur Pathophysiologie, Diagnostik und Therapie der KHK Stellung. Eine Parallelsitzung der Augsburger Tagung war Themen ausserhalb des Herz-Kreislaufgebietes gewidmet.

In diesem Heft konnte nur ein kleiner Teil der Vorträge und Poster publiziert werden. Weitere Vortragsmanuskripte sollten im "Normalverfahren» bei der Redaktion der Zeitschrift eingereicht werden.

Wir hoffen, dass das vorliegende Heft den wissenschaftlichen Ertrag der Augsburger Jahrestagung richtig wiedergibt, auch wenn wichtige Manuskripte aus Zeit- und Platzgründen (noch) nicht aufgenommen werden konnten. 